\title{
DUALITY WITHOUT CONSTRAINT QUALIFICATION FOR MULTIOBJECTIVE PROGRAMMING
}

\author{
R. R. EGUDO ${ }^{1}$, T. WEIR ${ }^{2}$ and B. MOND ${ }^{3}$
}

(Received 10 December 1990; revised 13 March 1991)

\begin{abstract}
Extending earlier duality results for multiobjective programs, this paper defines dual problems for convex and generalised convex multiobjective programs without requiring a constraint qualification. The duals provide multiobjective extensions of the classical duals of Wolfe and Schechter and some of the more recent duals of Mond and Weir.
\end{abstract}

\section{Introduction}

Ben-Israel, Ben-Tal and Zlobec [2] have given necessary and sufficient conditions for a vector to be an optimal solution of a convex (scalar) programming problem without the need for a constraint qualification. Mond and Zlobec [12] utilised the optimality conditions of [2] to define a dual problem to the (scalar) convex programming problem and established duality without the need for a constraint qualification. Earlier duality results of e.g. Wolfe [18] and Schechter [13] were shown to be special cases of the Mond and Zlobec duality formulation.

Some of the first-order results in [2] were extended to generalised convexity by Ben-Israel and Mond [3]. Following [3], Weir and Mond [16] defined dual problems for the scalar-valued programming problems where the usual convexity requirements for duality were relaxed and where a constraint qualification was not needed. Some of the previous duality results of Mond and Weir [11] involving generalised convex rather than convex functions were

\footnotetext{
ISchool of App. Science, Monash University College, Churchill VIC. 3842.

${ }^{2}$ Department of Mathematics, Australian Defence Force Academy, Campbell, ACT 2600.

${ }^{3}$ Department of Mathematics, La Trobe University, Bundoora, VIC 3083.

(C) Copyright Australian Mathematical Society 1992, Serial-fee code 0334-2700/92
} 
deduced as special cases of the dual formulations given in [16].

Characterisations of optimality without constraint qualification for multiobjective programming problems were developed first by Ben-Israel, Ben-Tal and Charnes [1]; their characterisations were presented in terms of systems of inequalities. Characterisations in terms of Lagrangians were developed by Zlobec in [19, 20]; Zlobec's Lagrangian characterisations are closely related to the classical scalarisation results in multiobjective programming of Geoffrion [7] and Karlin [8]. Based on Geoffrion's concept of proper efficiency and the scalar-valued programming optmality conditions of Ben-Israel, BenTal and Zlobec [2], Weir and Mond [17] have defined dual problems for the convex multiobjective programming problem and established duality without the need for a constraint qualification.

As in [17], the aim of this paper is to define dual problems for multiobjective programming problems where a constraint qualification is not assumed; however the approach here differs from that in [17] by focussing on efficiency rather than proper efficiency. This approach has the advantage of being suitable to define duals to nonconvex programming problems. The treatment here will provide multiobjective analogues of the classical duality results of Wolfe [18] and Schechter [13] and the more recent results of Mond and Weir [11].

\section{Notation and preliminaries}

Consider the multiobjective programming problem:

$$
\text { minimise }\left(f^{1}(x), f^{2}(x), \ldots, f^{q}(x)\right) \text { subject to } x \in F
$$

where $F \subset R^{n}$ and $f^{i}: R^{n} \rightarrow R, i \in Q=\{1,2, \ldots, q\}$. A point $x^{*} \in F$ is an efficient point if there is no other $x \in F$ such that $f^{i}(x) \leq f^{i}\left(x^{*}\right)$, $i \in Q$, with at least one strict inequality. For a fixed $r \in Q$ and $x^{*} \in R^{n}$ denote

$$
\begin{aligned}
& Q^{r}=Q \backslash\{r\}=\{k \in Q: k \neq r\} ; \\
& F^{r}\left(x^{*}\right)=\left\{x: f^{i}(x) \leq f^{i}\left(x^{*}\right), i \in Q^{r}\right\} \\
& Q^{r=}\left(x^{*}\right)=\left\{i \in Q^{r}: f^{i}(x)=f^{i}\left(x^{*}\right), \forall x \in F^{r}\left(x^{*}\right)\right\}, \\
& Q^{=}\left(x^{*}\right)=\bigcup_{r \in Q} Q^{r=}\left(x^{*}\right)=\left\{i \in Q: x \in F^{r}\left(x^{*}\right) \Rightarrow f^{i}(x)=f^{i}\left(x^{*}\right) \text { for some } r \in Q\right\} .
\end{aligned}
$$

For a function $f^{k}$, the cone of directions of constancy at $x^{*} \in R^{n}$ is

$$
D_{k}^{=}\left(x^{*}\right)=\left\{d: \exists T>0 \ni f^{k}\left(x^{*}+t d\right)=f^{k}\left(x^{*}\right), \forall t \in(0, T]\right\} \text {. }
$$


For an index set $K$ we denote

$$
\bigcap_{k \in K} D_{k}^{=}\left(x^{*}\right) \text { by } D_{K}^{=}\left(x^{*}\right) \text {. }
$$

For an arbitrary set $U \subset R^{n}$ its polar or dual is defined by

$$
U^{*}=\left\{u \in R^{n}: u^{\top} x \geq 0, \forall x \in U\right\}
$$

Now consider the multiobjective programming problem

$$
\begin{aligned}
& \text { minimise }\left(f^{1}(x), f^{2}(x), \ldots, f^{q}(x)\right) \\
& \text { subject to } g^{i}(x) \leq 0, i \in P=\{1,2, \ldots, p\} .
\end{aligned}
$$

$F$ will again denote the feasible set: $F=\left\{x: g^{i}(x) \leq 0, i \in P\right\}$. Let $x^{*} \in F$ and let $P\left(x^{*}\right)=\left\{i \in P: g^{i}\left(x^{*}\right)=0\right\}$ denote the index set of binding constraints at $x^{*}$. The minimal index set of binding constraints for $F$ is denoted by $P^{=}=\left\{i \in P: x \in F \Rightarrow g^{i}(x)=0\right\}$. We also denote

$$
P^{<}\left(x^{*}\right)=P\left(x^{*}\right) \backslash P^{=} \equiv\left\{i \in P\left(x^{*}\right): \exists x^{j} \in F \ni g^{i}\left(x^{j}\right)<0\right\} .
$$

Consider the problem (MP) where now all functions are assumed to be convex but not necessarily differentiable. The following lemma was originally proved for scalar valued mathematical programs [12], but as it does not depend on the objective function it remains valid for the multiobjective programming problem (MP).

LeMma 2.1. (a) The set $\left\{x \in R^{n}: g_{P^{=}}(x)=0\right\}$ is convex.

(b) If $x$ is feasible for (MP) and $g_{P^{-}}(u)=0$, then $(x-u)^{\top} y \geq 0$ for all $y \in\left[D_{p=}^{=}=(u)\right]^{*}$.

LEMMA 2.2. If $x$ is feasible for (MP), $g_{P^{*}}(u)=0$ and $f_{Q^{=}(u)}(x)=f_{Q^{-}(u)}(u)$ then $(x-u)^{\top} y \geq 0$ for all $y \in\left[D_{Q^{=}(u) \cup P^{=}}^{=}(u)\right]^{*}$.

Proof. Firstly observe that the set $\left\{x \in R^{n}: f_{Q^{=}(u)}(x)=f_{Q^{-}(u)}(u)\right\}$ may be redefined as $\left\{x \in R^{n}: h_{Q^{=}(u)}(u)=0\right\}$ where, for fixed $u, h_{Q^{\approx}(u)}(x)=$ $f_{Q^{=}(u)}(x)-f_{Q^{-}(u)}(u)$. Thus, from Lemma 2.1 , the set $\left\{x \in R^{n}: f_{Q^{-}(u)}(x)=\right.$ $\left.f_{Q^{\prime}(u)}(u)\right\}$ is convex. Therefore the set $\left\{x \in R^{n}: g_{P^{=}}(x)=0, f_{Q^{\prime}(u)}(x)=\right.$ $\left.f_{Q^{-}(u)}(u)\right\}$ is convex, being the intersection of two convex sets. Following [12], it is enough to show that

$$
\begin{aligned}
& g^{i}(\lambda x+(1-\lambda) u)=0, \\
& f^{j}(\lambda x+(1-\lambda) u)=0
\end{aligned}
$$


for all $0<\lambda<1, i \in P^{=}, j \in Q^{=}(u)$, because this implies that $(x-u) \in$ $D_{Q^{=}\left(x^{*}\right) \cup P^{=}}^{=}\left(x^{*}\right)$. If this were not true, then either $g^{i}\left(\lambda_{0} x+\left(1-\lambda_{0}\right) u\right)<0$, for some $0<\lambda_{0}<1$ and some $i \in P^{=}$; or $f^{j}\left(\lambda_{0} x+\left(1-\lambda_{0}\right) u\right)<0$, for some $0<\lambda_{0}<1$ and some $j \in Q^{=}(u)$. But this contradicts the convexity of the set

$$
\left\{x \in R^{n}: g_{P^{=}}(x)=0, f_{Q^{=}(u)}(x)=f_{Q^{=}(u)}(u)\right\} .
$$

The result follows from the definition of polars.

\section{Necessary and sufficient optimality conditions}

Consider the nonlinear programming problem

$$
\text { minimise } f^{0}(x) \text { subject to } g^{i}(x) \leq 0, i \in P \text {. }
$$

When $f^{0}$ and $g^{i}$ are scalar, not necessarily differentiable, convex functions, Ben-Israel, Ben-Tal and Zlobec [2] established the following necessary and sufficient conditions for a feasible point to be optimal in (SP).

THEOREM 3.1. The feasible point $x^{*}$ is an optimal solution for (SP) if and only if there exist scalars $y_{i} \geq 0, i \in P^{<}\left(x^{*}\right)$, such that

$$
\partial f^{0}\left(x^{*}\right)+\sum_{i \in P^{<}\left(x^{*}\right)} y_{i} \partial g^{i}\left(x^{*}\right) \in\left[D_{P^{*}}^{=}\left(x^{*}\right)\right]^{*} \text {. }
$$

For differentiable functions the convexity conditions of the above theorem may be weakened as shown by Weir and Mond [16].

Theorem 3.2. Let $f^{0}$ and $g^{i}(x) \leq 0, i \in P$, be pseudoconvex. The feasible point $x^{*}$ is an optimal solution for (SP) if and only if there exist scalars $y_{i} \geq 0, i \in P^{<}\left(x^{*}\right)$, such that

$$
\nabla f^{0}\left(x^{*}\right)+\sum_{i \in P^{<}\left(x^{*}\right)} y_{i} \nabla g^{i}\left(x^{*}\right) \in\left[D_{P^{*}}^{=}\left(x^{*}\right)\right]^{*} \text {. }
$$

Now consider the multi-objective program

$$
\begin{aligned}
& \text { minimise }\left(f^{1}(x), f^{2}(x), \ldots, f^{q}(x)\right) \\
& \text { subject to } g^{i}(x) \leq 0, i \in P .
\end{aligned}
$$

The following result is a well-known characterisation of the efficient solutions of (MP). 
LEMMA 3.3. A feasible solution $x^{*}$ in (MP) is efficient if and only if $x^{*}$ is optimal for each of the scalar optimisation problems:

$$
\begin{aligned}
& \text { minimise } f^{r}(x) \\
& \text { subject to } f^{j}(x) \leq f^{j}\left(x^{*}\right), j \in Q^{r}, \\
& g^{i}(x) \leq 0, i \in P
\end{aligned}
$$

for $r=1,2, \ldots, q$.

Theorem 3.1 and Lemma 3.3 can be used to derive necessary and sufficient optimality conditions without constraint qualification for the multiobjective programming problem (MP).

We firstly consider the case where all functions are assumed to be not necessarily differentiable convex functions.

THEOREM 3.4. The feasible point $x^{*}$ is an efficient solution for (MP) if and only if there exist scalars $\lambda_{i}^{*}>0, i \in Q$, with $\sum_{i \in Q} \lambda_{i}^{*}=1$ and $y_{i}^{*} \geq 0$, $i \in P^{<}\left(x^{*}\right)$, such that

$$
\sum_{i \in Q} \lambda_{i}^{*} \partial f^{i}\left(x^{*}\right)+\sum_{i \in P^{<}\left(x^{*}\right)} y_{i}^{*} \partial g^{i}\left(x^{*}\right) \in\left[D_{Q^{*}\left(x^{*}\right) \cup P^{*}}^{=}\left(x^{*}\right)\right]^{*} .
$$

Proof. (Necessity) Since $x^{*}$ is efficient for (MP), it follows from Lemma 3.3 that $x^{*}$ is optimal for each of the scalar nonlinear programming problems $\left(P^{r}\left(x^{*}\right)\right), r \in Q$. Applying Theorem 3.1 to each $\left(P^{r}\left(x^{*}\right)\right), r \in Q$, gives the existence of scalars $\lambda_{r i}>0$, and $y_{r i} \geq 0, i \in P^{<}\left(x^{*}\right)$, such that

$$
\partial f^{r}\left(x^{*}\right)+\sum_{i \in Q^{r} \backslash Q^{r=}\left(x^{*}\right)} \lambda_{r i} \partial f^{i}\left(x^{*}\right)+\sum_{i \in P^{<}\left(x^{*}\right)} y_{r i} \partial g^{i}\left(x^{*}\right) \in\left[D_{Q^{r=}\left(x^{*}\right) \cup P^{*}}^{=}\left(x^{*}\right)\right]^{*}
$$

for each $r \in Q$. Now summing the above over $r \in Q$, scaling appropriately, and using the properties of polars implies the necessary condition.

(Sufficiency) Suppose there exists scalars $\lambda_{i}^{*}>0, i \in Q$, and $y_{i}^{*} \geq 0$, $i \in P^{<}\left(x^{*}\right)$, such that (3.1) is satisfied but that $x^{*}$ is not efficient for (MP). Then there exist $v_{i} \in \partial f^{i}\left(x^{*}\right), i=1,2, \ldots, q, z_{i} \in \partial g^{i}\left(x^{*}\right), i \in P^{<}\left(x^{*}\right)$ and $d \in\left[D_{Q^{-}\left(x^{*}\right) \cup P^{=}}^{=}\left(x^{*}\right)\right]^{*}$ such that

$$
\sum_{i \in Q} \lambda_{i}^{*} v_{i}+\sum_{i \in P^{<}\left(x^{*}\right)} y_{i}^{*} z_{i}=d
$$

and there exists $u \in F$ such that

$$
\begin{array}{ll}
f^{i}(u)<f^{i}\left(x^{*}\right) & \text { for some } i, \text { and } \\
f^{j}(u) \leq f^{j}\left(x^{*}\right) & \text { for all } j \neq i .
\end{array}
$$


It follows that

$$
\sum_{i \in Q} \lambda_{i}^{*} f^{i}(u)<\sum_{i \in Q} \lambda_{i}^{*} f^{i}\left(x^{*}\right)
$$

By convexity

$$
\begin{aligned}
\sum_{i \in Q} \lambda_{i}^{*} f^{i}(u)-\sum_{i \in Q} \lambda_{i}^{*} f^{i}\left(x^{*}\right) \\
\quad \geq\left(u-x^{*}\right)^{\top} \sum_{i \in Q} \lambda_{i}^{*} v_{i} \\
\quad=\left(u-x^{*}\right)^{\top}\left(d-\sum_{i \in P^{<}\left(x^{*}\right)} y_{i}^{*} z_{i}\right) \\
\quad \geq-\left(u-x^{*}\right)^{\top} \sum_{i \in P^{<}\left(x^{*}\right)} y_{i}^{*} z_{i} \text { by Lemma } 2.2 \\
\quad \geq-\sum_{i \in P^{<}\left(x^{*}\right)} y_{i}^{*} g^{i}(u)+\sum_{i \in P^{<}\left(x^{*}\right)} y_{i}^{*} g^{i}\left(x^{*}\right) \text { by convexity } \\
\quad=-\sum_{i \in P^{<}\left(x^{*}\right)} y_{i}^{*} g^{i}(u) \text { by definition of } P^{<}\left(x^{*}\right) \\
\quad \geq 0 \text { since } u \text { is feasible for (MP). }
\end{aligned}
$$

This clearly contradicts (3.2); hence $x^{*}$ is efficient. Although the above theorem may be specialised to differentiable convex functions (by replacing subgradients with gradients), a stronger result for problems involving differentiable functions is available. Assume now that all functions appearing in (MP) are differentiable pseudoconvex functions.

THEOREM 3.5. The feasible point $x^{*}$ is an efficient solution for (MP) if and only if there exists scalars $\lambda_{i}^{*}>0, i \in Q$, with $\sum_{i \in Q} \lambda_{i}^{*}=1$ and $y_{i}^{*} \geq 0$, $i \in P^{<}\left(x^{*}\right)$, such that

$$
\sum_{i \in Q} \lambda_{i}^{*} \nabla f^{i}\left(x^{*}\right)+\sum_{i \in P^{<}\left(x^{*}\right)} y_{i}^{*} \nabla g^{i}\left(x^{*}\right) \in\left[D_{Q^{-}\left(x^{*}\right) \cup P^{*}}^{=}\left(x^{*}\right)\right]^{*} .
$$

Proof. (Necessity) This follows in exactly the same manner as for Theorem 3.5 but using Theorem 3.2 rather than Theorem 3.1 .

(Sufficiency) Suppose there exist scalars $\lambda_{i}^{*}>0, i \in Q$, and $y_{i}^{*} \geq 0$, $i \in P^{<}\left(x^{*}\right)$, such that (3.3) is satisfied but that $x^{*}$ is not efficient for (MP). Then there exists $u \in F$ such that

$$
\begin{array}{ll}
f^{i}(u)<f^{i}\left(x^{*}\right) & \text { for some } i, \text { and } \\
f^{j}(u) \leq f^{j}\left(x^{*}\right) & \text { for all } j \neq i .
\end{array}
$$


The pseudoconvexity of each $f^{i}$ implies that

$$
\begin{array}{ll}
\left(u-x^{*}\right)^{\top} \nabla f^{i}\left(x^{*}\right)<0 & \text { for } i, \text { and } \\
\left(u-x^{*}\right)^{\top} \nabla f^{j}\left(x^{*}\right) \leq 0 & \text { for all } j \neq i .
\end{array}
$$

Multiplying each of these inequalities by $\lambda_{i}^{*}$ and summing over all $i \in Q$ gives

$$
\left(u-x^{*}\right)^{\top} \sum_{i \in Q} \lambda_{i}^{*} \nabla f^{i}\left(x^{*}\right)<0 .
$$

Therefore, by (3.2) and Lemma 2.2,

$$
\left(u-x^{*}\right)^{\top} \sum_{i \in P^{<}\left(x^{*}\right)} y_{i}^{*} \nabla g^{i}\left(x^{*}\right)>0 .
$$

For $i \in P^{<}\left(x^{*}\right), g^{i}(u)-g^{i}\left(x^{*}\right) \leq 0$ and since each $g^{i}$ is pseudoconvex and hence quasiconvex, it follows that

$$
\left(u-x^{*}\right)^{\top} \nabla g^{i}\left(x^{*}\right) \leq 0 .
$$

Thus

$$
\left(u-x^{*}\right)^{\top} \sum_{i \in P^{<}\left(x^{*}\right)} y_{i}^{*} \nabla g^{i}\left(x^{*}\right) \leq 0 .
$$

This is a contradiction to $(3.4)$; hence $x^{*}$ is efficient.

REMARKs. (i) If the constraints of problem (MP) satisfy the Slater constraint qualification, then $P^{=}$is empty. In this case the optimality conditions simplify as the constraints of (MP) do not appear in the dual cones in (3.1) and (3.3).

(ii) If further, the constraints of $\left(P^{r}\left(x^{*}\right)\right)$ satisfy the Slater constraint qualification, then the dual cones in (3.1) and (3.3) contain only the zero vector. In this case the optimality conditions reduce to those of Geoffrion [7] in the convex case (and $x^{*}$ is properly efficient) and to those of Weir [15] for the pseudoconvex case (in this case $x^{*}$ satisfies Kuhn and Tucker's [9] definition of proper efficiency).

(iii) Note that if $q=1$, then the optimality conditions of Theorems 3.4 and 3.5 reduce to those of Ben-Israel, Ben-Tal and Zlobec [2] and Weir and Mond [16] respectively.

(iv) Consider the special case where the functions $f^{i}, i \in Q$, are pseudolinear, i.e. pseudoconvex and pseudoconcave. Note that for $i \in Q^{=}\left(x^{*}\right)$,

$$
\begin{aligned}
D_{i}^{=}\left(x^{*}\right) & =\left\{d: \exists T>0 \ni f^{i}\left(x^{*}+t d\right)=f^{i}\left(x^{*}\right), \forall t \in(0, T]\right\} \\
& =\left\{d: \nabla f^{i}\left(x^{*}\right)^{\top} d=0\right\} \\
& =\left\{d: \nabla f^{i}\left(x^{*}\right)^{\top} d \leq 0\right\} \quad \text { by Lemma } 3.9(\mathrm{e}) \text { of }[2] .
\end{aligned}
$$


Thus

$$
\left[D_{i}^{=}\left(x^{*}\right)\right]^{*}=\left\{-\lambda_{i} \nabla f^{i}\left(x^{*}\right): \lambda_{i} \geq 0\right\}, \quad i \in Q^{=}\left(x^{*}\right),
$$

and, since each $D_{i}^{=}\left(x^{*}\right)$ is polyhedral (see e.g. [2]),

$$
\left[D_{Q^{=}\left(x^{*}\right)}^{=}\left(x^{*}\right)\right]^{*}=\left\{-\sum_{i \in Q^{=}\left(x^{*}\right)} \lambda_{i} \nabla f^{i}\left(x^{*}\right): \lambda_{i} \geq 0\right\} .
$$

The optimality condition appearing in Theorem 3.5 may then be expressed as: there exist scalars $\lambda_{i}^{*}>0, i \in Q, \lambda_{i} \geq 0, i \in Q^{=}\left(x^{*}\right)$, and $y_{i}^{*} \geq 0$, $i \in P^{<}\left(x^{*}\right)$, such that

$$
\sum_{i \in Q} \lambda_{i}^{*} \nabla f^{i}\left(x^{*}\right)+\sum_{i \in P^{<}\left(x^{*}\right)} y_{i}^{*} \nabla g^{i}\left(x^{*}\right)+\sum_{i \in Q^{*}\left(x^{*}\right)} \lambda_{i} \nabla f^{i}\left(x^{*}\right) \in\left[D_{P^{=}}^{=}\left(x^{*}\right)\right]^{*} .
$$

That is, there exist scalars $\mu_{i}>0, i \in Q$, and $y_{i}^{*} \geq 0, i \in P^{<}\left(x^{*}\right)$, such that

$$
\sum_{i \in Q} \mu_{i} \nabla f^{i}\left(x^{*}\right)+\sum_{i \in P^{<}\left(x^{*}\right)} y_{i}^{*} \nabla g^{i}\left(x^{*}\right) \in\left[D_{P^{=}}^{=}\left(x^{*}\right)\right]^{*} .
$$

If in addition the functions $g^{i}, i \in P$, are also pseudolinear, then the optimality conditions may be expressed as: there exist scalars $\mu_{i}>0, i \in Q$, and $y_{i} \geq 0, i \in P\left(x^{*}\right)$ such that

$$
\sum_{i \in Q} \mu_{i} \nabla f^{i}\left(x^{*}\right)+\sum_{i \in P\left(x^{*}\right)} y_{i} \nabla g^{i}\left(x^{*}\right)=0 .
$$

The latter condition has been derived in Chew and Choo [4]; note that the condition on the constraints $g^{i}$ is the weak reverse convex constraint qualification [10].

\section{Duality}

Based on the optimality condition without constraint qualification given in Theorem 3.5, we may relate (MP) to the following dual problem:

$$
\text { maximise }\left(f^{1}(u)+y^{\top} g(u), \ldots, f^{q}(u)+y^{\top} g(u)\right)
$$

subject to

$$
\sum_{i \in Q} \lambda_{i} \partial f^{i}(u)+\sum_{i \in P} y_{i} \partial g^{i}(u) \in\left[D_{Q^{-}(u) \cup P^{=}}^{=}(u)\right]^{*}
$$




$$
\begin{aligned}
& y_{i} \geq 0, \quad i=1,2, \ldots, p, \\
& \lambda_{i}>0, \quad i=1,2, \ldots, q, \\
& g_{P=}(u)=0, \\
& \sum_{i \in Q} \lambda_{i}=1 .
\end{aligned}
$$

Here the functions $f^{i}, i \in Q$, and $g^{i}, i \in P$, are scalar, not necessarily differentiable convex functions. Let $H$ denote the set of feasible solutions to (D1).

Theorem 4.1 (Weak Duality). Let $x \in F$ and $(u, \lambda, y) \in H$. Then the following cannot occur.

$$
\begin{aligned}
& f^{i}(x)<f^{i}(u)+y^{\top} g(u) \text { for some } i, \text { and } \\
& f^{j}(x) \leq f^{j}(u)+y^{\top} g(u) \text { all } j \neq i .
\end{aligned}
$$

Proof. There exist $v_{i} \in \partial f^{i}(u), i=1,2, \ldots, q ; z_{i} \in \partial g^{i}(u), i=$ $1,2, \ldots, p$, and $d \in\left[D_{Q^{=}(u) \cup P^{-}}^{=}(u)\right]^{*}$ such that

$$
\sum_{i \in Q} \lambda_{i} v_{i}+\sum_{i \in P} y_{i} z_{i}=d
$$

Now

$$
\begin{aligned}
\sum_{i \in Q} \lambda_{i} & {\left[f^{i}(x)-\left(f^{i}(u)+y^{\top} g(u)\right)\right]=\sum_{i \in Q} \lambda_{i} f^{i}(x)-\sum_{i \in Q} \lambda_{i} f^{i}(u)-y^{\top} g(u) } \\
& \geq(x-u)^{\top} \sum_{i \in Q} \lambda_{i} v_{i}-y^{\top} g(u) \\
& =-(x-u)^{\top} \sum_{i \in Q} y_{i} z_{i}-y^{\top} g(u)+(x-u)^{\top} d \\
& \geq \sum_{i \in P} y_{i}\left[g^{i}(u)-g^{i}(x)\right]-y^{\top} g(u) \\
& =-y^{\top} g(x) \geq 0 .
\end{aligned}
$$

This shows that for feasible $x$ of (MP) and $(u, \lambda, y)$ of (D1)

$$
\sum_{i \in Q} \lambda_{i}\left[f^{i}(x)-\left(f^{i}(u)+y^{\top} g(u)\right)\right] \geq 0 .
$$

If, for some $x$ feasible for (MP) and $(u, \lambda, y)$ feasible for (D1),

$$
\begin{array}{ll}
f^{i}(x)<f^{i}(u)+y^{\top} g(u) & \text { for some } i, \text { and } \\
f^{j}(x) \leq f^{j}(u)+y^{\top} g(u) & \text { all } j \neq i
\end{array}
$$


then

$$
\sum_{i \in Q} \lambda_{i}\left[f^{i}(x)-\left(f^{i}(u)+y^{\top} g(u)\right)\right]<0,
$$

which is a contradiction to (4.1).

THEOREM 4.2 (Strong Duality). If $x^{*}$ is efficient for (MP) then there exist $y_{i}^{*} \geq 0, i=1,2, \ldots, p ; \lambda_{i}^{*}>0, i=1,2, \ldots, q$ such that $\left(x^{*}, y_{i}^{*}, i=\right.$ $\left.1,2, \ldots, p, \lambda_{i}^{*}, i=1,2, \ldots, q\right)$ is efficient for (D1) and the objective values of (MP) and (D1) are equal.

Proof. If $x^{*}$ is efficient for (MP) then by Theorem 3.4 there exist $\lambda_{i}^{*}>0$, $i \in Q$, and $y_{i} \geq 0, i \in P^{<}\left(x^{*}\right)$, and $d \in\left[D_{Q^{-}\left(x^{*}\right) \cup P^{*}}^{=}\left(x^{*}\right)\right]^{*}$ such that (3.1) is satisfied. Setting $y_{i}^{*}=0$ for $i \notin P^{<}\left(x^{*}\right)$ implies that $\left(x^{*}, y_{i}^{*}, i \in\right.$ $\left.P, \lambda_{i}^{*}, i \in Q\right)$ is feasible for (D1). If $\left(x^{*}, y_{i}^{*}, i \in P, \lambda_{i}^{*}, i \in Q\right)$ was not efficient for (D1) there would exist $(u, y, \lambda)$ feasible for (D1) such that

$$
\begin{array}{ll}
f^{i}(u)+y^{\top} g(u)>f^{i}\left(x^{*}\right)+y^{* \top} g\left(x^{*}\right) & \text { for some } i, \text { and } \\
f^{j}(u)+y^{\top} g(u) \geq f^{j}\left(x^{*}\right)+y^{* \top} g\left(x^{*}\right) & \text { all } j \neq i ;
\end{array}
$$

however, since $y^{* t} g\left(x^{*}\right)=0$, this would contradict weak duality. The objectives of (MP) and (D1) are clearly equal at their respective efficient points.

REMARKs. (i) If the constraints of (MP) satisfy the Slater constraint qualification, then $P^{=}$is empty. The dual problem (D1) then reduces to

$$
\begin{aligned}
& \text { maximise }\left(f^{1}(u)+y^{\top} g(u), \ldots, f^{q}(u)+y^{\top} g(u)\right) \\
& \text { subject to }
\end{aligned}
$$

$$
\begin{gathered}
\sum_{i \in Q} \lambda_{i} \partial f^{i}(u)+\sum_{i \in P} y_{i} \partial g^{i}(u) \in\left[D_{Q^{=}(u)}^{=}(u)\right]^{*}, \\
y_{i} \geq 0, \quad i=1,2, \ldots, p \\
\lambda_{i}>0, \quad i=1,2, \ldots, q, \\
\sum_{i \in Q} \lambda_{i}=1 .
\end{gathered}
$$

If additionally, for each $u, Q^{=}(u)$ is empty, then the results of [17] are recovered; however duality must then be expressed in terms of proper efficiency.

(ii) If the functions $f^{i}$ and $g^{i}$ are differentiable then subgradients may simply be replaced in (D1) and (D2) by gradients. In the case of $Q^{=}(u) \cup P^{=}$ being empty, the duality results of [5], [6] and [14] are recovered. 
(iii) Note that if $q=1$, then (D1) is the Mond and Zlobec dual to the scalar nonlinear programming problem. In this case, if the constraints of (MP) satisfy the Slater condition, the dual becomes the formulation due to Schechter [13]; if the functions are differentiable then Wolfe's [18] duality results are recovered.

We will now construct a dual problem and develop duality theorems without constraint qualification for (MP) where the functions of (MP) are not assumed convex but are differentiable. Consider the problem,

$$
\begin{gathered}
\text { maximise }\left(f^{1}(u), \ldots, f^{q}(u)\right) \\
\text { subject to } \\
\sum_{i \in Q} \lambda_{i} \nabla f^{i}(u)+\sum_{i \in P} y_{i} \nabla g^{i}(u) \in\left[D_{Q^{-}(u) \cup P^{=}}^{=}(u)\right]^{*}, \\
y_{i} \geq 0, \quad i=1,2, \ldots, p, \\
\lambda_{i}>0, \quad i=1,2, \ldots, q, \\
g_{P}=(u)=0, \\
y_{i} g_{i}(u) \geq 0, \quad i=1,2, \ldots, p, \\
\sum_{i \in Q} \lambda_{i}=1 .
\end{gathered}
$$

Here the functions $f^{i}, i \in Q$, and $g^{i}, i \in P$, are scalar, pseudoconvex functions. $H$ will denote the set of feasible solutions to (D3).

Theorem 4.3 (Weak Duality). Let $x \in F$ and $(u, \lambda, y) \in H$. Then the following cannot occur.

$$
\begin{aligned}
& f^{i}(x)<f^{i}(u) \text { for some } i, \text { and } \\
& f^{j}(x) \leq f^{j}(u) \text { all } j \neq i .
\end{aligned}
$$

Proof. For $i \in P$,

$$
y_{i} g^{i}(x)-y_{i} g^{i}(u) \leq 0 .
$$

Since each $g^{i}$ is pseudoconvex and hence quasiconvex, it follows that

$$
(x-u)^{\top} y_{i} \nabla g^{i}(u) \leq 0
$$

and so

$$
(x-u)^{\top} \sum_{i \in P} y_{i} \nabla g^{i}(u) \leq 0 .
$$

From the constaints of (D3), it follows for some $d \in\left[D_{Q^{=}(u) \cup P}^{=}(u)\right]^{*}$ that

$$
(x-u)^{\top} \sum_{i \in Q} \lambda_{i} \nabla f^{i}(u) \geq(x-u)^{\top} d
$$


and by Lemma 2.2

$$
(x-u)^{\top} \sum_{i \in Q} \lambda_{i} \nabla f^{i}(u) \geq 0 .
$$

If for some $x$ feasible for (MP) and $(u, \lambda, y)$ feasible for (D2)

$$
\begin{aligned}
& f^{i}(x)<f^{i}(u) \quad \text { for some } i, \text { and } \\
& f^{j}(x) \leq f^{j}(u) \quad \text { all } j \neq i .
\end{aligned}
$$

then

$$
(x-u)^{\top} \sum_{i \in Q} \lambda_{i} \nabla f^{i}(u)<0 .
$$

This is a contradiction to (4.2).

TheOREM 4.4 (Strong Duality). If $x^{*}$ is efficient for (MP) then there exist $y_{i}^{*} \geq 0, i=1,2, \ldots, p ; \lambda_{i}^{*}>0, i=1,2, \ldots, q$, such that $\left(x^{*}, y_{i}^{*}, i=\right.$ $\left.1,2, \ldots, p ; \lambda_{i}^{*}, i=1,2, \ldots, q\right)$ is efficient for (D3) and the objective values of (MP) and (D3) are equal.

Proof. If $x^{*}$ is efficient for (MP) then by Theorem 3.4 there exists $\lambda_{i}^{*}>0$, $i \in Q$, and $y_{i} \geq 0, i \in P^{<}\left(x^{*}\right)$, and $d \in\left[D_{Q^{-}\left(x^{*}\right) \cup P^{*}}^{=}\left(x^{*}\right)\right]^{*}$ such that (3.1) is satisfied. Setting $y_{i}^{*}=0$ for $i \notin P^{<}\left(x^{*}\right)$ implies that $\left(x^{*}, y_{i}^{*}, i \in\right.$ $\left.P, \lambda_{i}^{*}, i \in Q\right)$ is feasible for (D3). If $\left(x^{*}, y_{i}^{*}, i \in P, \lambda_{i}^{*}, i \in Q\right)$ was not efficient for (D3) there would exist $(u, y, \lambda)$ feasible for (D3) such that

$$
\begin{aligned}
& f^{i}(u)>f^{i}\left(x^{*}\right) \quad \text { for some } i, \text { and } \\
& f^{j}(u) \geq f^{j}\left(x^{*}\right) \text { all } j \neq i
\end{aligned}
$$

however, since $x^{*}$ is feasible for (MP), this would contradict weak duality. The objectives of (MP) and (D3) are clearly equal at their respective efficient points.

REMARKS. (i) If the constraints of (MP) satisfy the Slater constraint qualification, then $P^{=}$is empty. The dual problem (D3) then reduces to 


$$
\text { maximise }\left(f^{1}(u), \ldots, f^{q}(u)\right)
$$

subject to

$$
\begin{aligned}
& \sum_{i \in Q} \lambda_{i} \nabla f^{i}(u)+\sum_{i \in P} y_{i} \nabla g^{i}(u) \in\left[D_{Q^{-}(u)}^{=}(u)\right]^{*} \\
& y_{i} \geq 0, \quad i=1,2, \ldots, p \\
& \lambda_{i}>0, \quad i=1,2, \ldots, q, \\
& y_{i} g^{i}(u) \geq 0, \quad i=1,2, \ldots, p \\
& \sum_{i \in Q} \lambda_{i}=1 .
\end{aligned}
$$

If additionally, for each $u, Q^{=}(u)$ is empty, then the results of Egudo [5, 6] and Weir [14] are recovered; duality in [14] was expressed in terms of proper efficiency.

(ii) Note that if $q=1$, then (D3) is the formulation of the Weir and Mond [16] dual to the scalar nonlinear programming problem. In this case, if the constraints of (MP) satisfy the Slater condition, the dual becomes a variant of the formulation due to Mond and Weir [11].

(iii) Note that in the special case where the functions $f^{i}, i=1,2, \ldots, q$, are pseudolinear the dual (D3) may be expressed as

$$
\begin{aligned}
& \text { maximise }\left(f^{1}(u), \ldots, f^{q}(u)\right) \\
& \text { subject to } \\
& \sum_{i \in Q} \lambda_{i} \nabla f^{i}(u)+\sum_{i \in P} y_{i} \nabla g^{i}(u) \in\left[D_{P^{*}}^{=}(u)\right]^{*}, \\
& y^{i} \geq 0, \quad i=1,2, \ldots, p, \\
& \lambda_{i}>0, \quad i=1,2, \ldots, q, \\
& y_{i} g^{i}(u) \geq 0, \quad i=1,2, \ldots, p \\
& \sum_{i \in Q} \lambda_{i}=1 .
\end{aligned}
$$

If, in addition, the functions $g^{i}$ are pseudolinear the dual reduces to that given in Weir [15].

(iv) Note that the dual (D3) and Theorems 4.3 and 4.4 are directly applicable to multiobjective fractional programming problems where the objectives take the form $f^{i}=k^{i} / h^{i}$ where $k^{i}$ and $-h^{i}$ are differentiable convex functions (and hence $f^{i}$ is pseudoconvex). 


\section{References}

[1] A. Ben-Israel, A. Ben-Tal and A. Charnes, "Necessary and sufficient conditions for Pareto Optimum in convex programming", Econometrica 45 (1977) 811-820.

[2] A. Ben-Israel, A. Ben-Tal and S. Zlobec, Optimality in nonlinear programming. a feasible directions approach, (Wiley-Interscience, New York, 1981).

[3] A. Ben-Israel and B. Mond, "First order optimality conditions for generalized convex programming: a feasible directions approach", Utilitas Mathematica 25 (1984) 249-262.

[4] K. L. Chew and E. U. Choo, "Pseudolinearity and efficiency", Mathematical Programming 28 (1982) 226-239.

[5] R. R. Egudo, “Efficiency and generalized convex duality for multiobjective programs", J. Math. Anal. Appl. 138 (1989) 84-94.

[6] R. R. Egudo, “Multiobjective duality in mathematical programming”, PhD. Thesis, La Trobe University, Bundoora, Victoria, Australia, 1990.

[7] A. M. Geoffrion, "Proper efficiency and the Theory of Vector Maximization", J. Math. Anal. Appl. 22 (1968) 618-630.

[8] S. Karlin, Mathematical methods and theory in games, programming and economics, Vol I, (Addison-Wesley, Reading Massachusetts, 1959).

[9] H. W. Kuhn and A. W. Tucker, "Nonlinear programming", in Proceedings of the Second Berkeley Symposium on Mathematical Statistics and Probability, (ed. J. Neyman), (University of California Press, Berkeley, California, 1951), 481-493.

[10] O. L. Mangasarian, Nonlinear programming, (McGraw-Hill, New York, 1969).

[11] B. Mond and T. Weir, "Generalized concavity and duality", in Generalized concavity in optimization and economics, (eds. S. Schaible and W. T. Ziemba), (Academic Press, N.Y. 1981), 263-279.

[12] B. Mond and S. Zlobec, "Duality for nondifferentiable programming without a constraint qualification", Utilitas Mathematica 15 (1979) 291-302.

[13] M. Schechter, “A subgradient duality theorem”, J. Math. Anal. Appl. 61 (1977) 850-855.

[14] T. Weir, "Proper efficiency and duality for vector valued optimization problems", $J$. Austral. Math. Soc. (Series A) 43 (1987) 21-35.

[15] T. Weir, "On efficiency, proper efficiency and duality in multiobjective programming", Asia Pacific J. of Operations Research 7 (1990) 46-54.

[16] T. Weir and B. Mond, "Duality for generalized convex programming without a constraint qualification", Utilitas Mathematica 31 (1987) 233-242.

[17] T. Weir and B. Mond, "Multiple objective programming duality without a constraint qualification", Utilitas Mathematica 39 (1991) 41-55.

[18] P. Wolfe, “A duality theorem for nonlinear programming”, Quart. Appl. Math. 19 (1961) 239-244.

[19] S. Zlobec, "Two characterizations of Pareto Minima in convex multicriteria optimization", Aplikace Matermatiky 29 (1984) 342-349.

[20] S. Zlobec, "Input optimization: III. Optimal realizations of multi-objective models", Optimization 17 (1986) 429-445. 